\title{
Genetic and Environmental Factors Influencing the Production and Distribution of Protoperithecia in Sordaria brevicollis
}

\author{
By D. J. MACDONALD AND D. J. BOND \\ Institute of Animal Genetics, University of Edinburgh, Edinburgh EH9 $3 \mathrm{JN}$
}

(Received 27 October 1975; revised 29 December 1975)

\begin{abstract}
SUMMARY
Fertile cultures of Sordaria brevicollis have been shown to make incipient fruiting bodies (protoperithecia) only after contact with the edge of the containing vessel. Experiments with different sizes of Petri dish and different amounts of media confirmed that contact with the edge was the stimulus for fruiting. Mutants with an altered response to the edge were studied. A correlation between the glucose 6-phosphate concentration in the mycelium and the production and distribution of protoperithecia was demonstrated, but there was no evidence that this was causal.
\end{abstract}

\section{INTRODUCTION}

In ascomycete fungi such as Sordaria brevicollis, the incipient fruiting bodies (protoperithecia) are produced on and from the vegetative mycelium. After fertilization by a strain of opposite mating type, the protoperithecia develop into flask-shaped perithecia which contain the asci and ascospores. Morphogenetic systems of this type have been the subject of extensive analyses (reviewed by Hawker, 1966); these have revealed on array of environmental conditions which bring about a switch from the vegetative to the reproductive state.

A particularly interesting stimulus for fruiting was first described in Pyronema confluens by Robinson (1926). It was clearly established that fruiting commenced after the mycelium of a colony had reached the edge of the containing vessel. Apparently the presence of a growing mycelial front inhibited the differentiation of fruiting bodies. Inoue \& Furuya (1970) have described a similar phenomenon in Gelasinospora reticulospora, although in this case there was an interaction between light and the two different growth states.

In addition to affecting the formation of fruiting bodies, the edge of the containing vessel has also been shown to influence their distribution. In Chaetomium globosum, perithecia are formed before the colony reaches the edge but they are most numerous at the edge of the colony nearest to the Petri dish wall (Buston \& Rickard, 1956). This was attributed to the increased concentration of organic phosphates at the plate edge. Sugar phosphates, such as glucose 6-phosphate, had previously been shown to stimulate protoperithecial production in Melanospora when added to the medium (Hawker, 1948).

We have discovered that an edge effect, similar to that demonstrated in Pyronema, exists $\mathrm{n}$ Sordaria brevicollis. Protoperithecial production commenced all over the colony after the mycelial front had reached the edge of the containing vessel. 


\section{METHODS}

Crossing procedure. All crosses of Sordaria brevicollis were carried out using a standardized procedure. When this was adopted the phenotype of the crosses, defined by the distribution of protoperithecia on the mycelium, was a highly reproducible feature of any isolate.

Crosses were carried out on a standard volume $(20 \mathrm{ml})$ of corn meal agar containing (g/l distilled water): Difco corn meal agar, $17 \mathrm{~g}$; sucrose, $3 \mathrm{~g}$; glucose, $2 \mathrm{~g}$; yeast extract, I g. Male and female components were inoculated in separate Petri dishes and incubated for 4 days at $25^{\circ} \mathrm{C}$. Microconidia from the male parent were then harvested in sterile distilled water, and filtered through two layers of sterile muslin to remove mycelial fragments and protoperithecia. The female parent was fertilized with a measured volume (usually $2 \mathrm{ml}$ ) of the microconidial suspension.

The distribution of perithecia after further incubation accurately reflected the distribution of protoperithecia (unpublished results). There was no evidence for differential fertilization. A culture was fertilized merely to facilitate determination of its crossing phenotype.

Determination of glucose 6-phosphate. The concentration of glucose 6-phosphate per $\mathrm{g}$ wet wt mycelium was determined spectrophotometrically. Mycelium from cultures grown at $25^{\circ} \mathrm{C}$ on the surface of a dialysis membrane was homogenized in 10 vols ice-cold trichloroacetic acid solution $(5 \%, \mathrm{w} / \mathrm{v})$, and centrifuged. The supernatant was extracted five times with ether to remove the trichloroacetic acid. To $2 \cdot \mathrm{I} \mathrm{ml}$ extracted supernatant were added $0.8 \mathrm{ml}$ buffer ( $0.4 \mathrm{M}$-glycylglycine, $0.15 \mathrm{M}-\mathrm{MgCl}_{2}, 0 . \mathrm{I} \mathrm{M}$-glucose; $\mathrm{pH} 7.4$ ) and $0 . \mathrm{I} \mathrm{ml} 30 \mathrm{nM}-$ NADP. On addition of 3 units of glucose 6-phosphate dehydrogenase, the change in absorbance at $340 \mathrm{~nm}$ was measured and compared with a standard curve to establish the concentration of glucose 6-phosphate in the sample.

The relationship of any particular colony to the edge was determined from its growth rate. Usually, whole colonies were homogenized; but in some experiments colonies that were $60 \mathrm{~mm}$ or more in diameter were divided, the margin and centre being determined separately. In these cases a marginal zone, approximately $15 \mathrm{~mm}$ wide, was cut off whilst the colony was still attached to the dialysis membrane.

\section{RESULTS AND DISCUSSION \\ Genetic segregations affecting crossing phenotype}

Segregations were discovered (to be fully described elsewhere) at two loci which we have called perithecial- $I$ and perithecial-2. These genes have a pronounced effect on the formation and distribution of protoperithecia. Of the four possible allelic combinations, three are relevant to this paper. per- $I^{+}$per- $-2^{+}$is the wild-type condition, where protoperithecia are distributed more or less evenly across the Petri dish. per- $I^{-}$per-2- is female sterile and completely devoid of protoperithecia. per $-\Gamma^{-}$per- $2^{+}$is fertile with a characteristic crossing phenotype; protoperithecia are produced in a concentrated ring just in from the edge of the dish (Fig. I $a$ ).

To carry out the medium transfer experiments described below, cultures were grown on sterile dialysis membranes placed on the surface of corn meal agar. This modification of the crossing technique did not affect the sterility of per- $I^{-}$per-2- $2^{-}$, but it did increase the number of protoperithecia in per- $I^{+}$per-2- $2^{-}$and per- $I^{+}$per-2 $2^{+}$cultures. However, there was a striking change in the crossing phenotype of per- $I^{-}$per- $2^{+}$cultures (Fig. I $b$ ). The distinct margin to protoperithecial production, and the peripheral zone more or less completely devoid of protoperithecia, were highly reproducible features of per- $\mathrm{I}^{-}$per-2 $\mathbf{2}^{+}$cultures crossed under these conditions. 

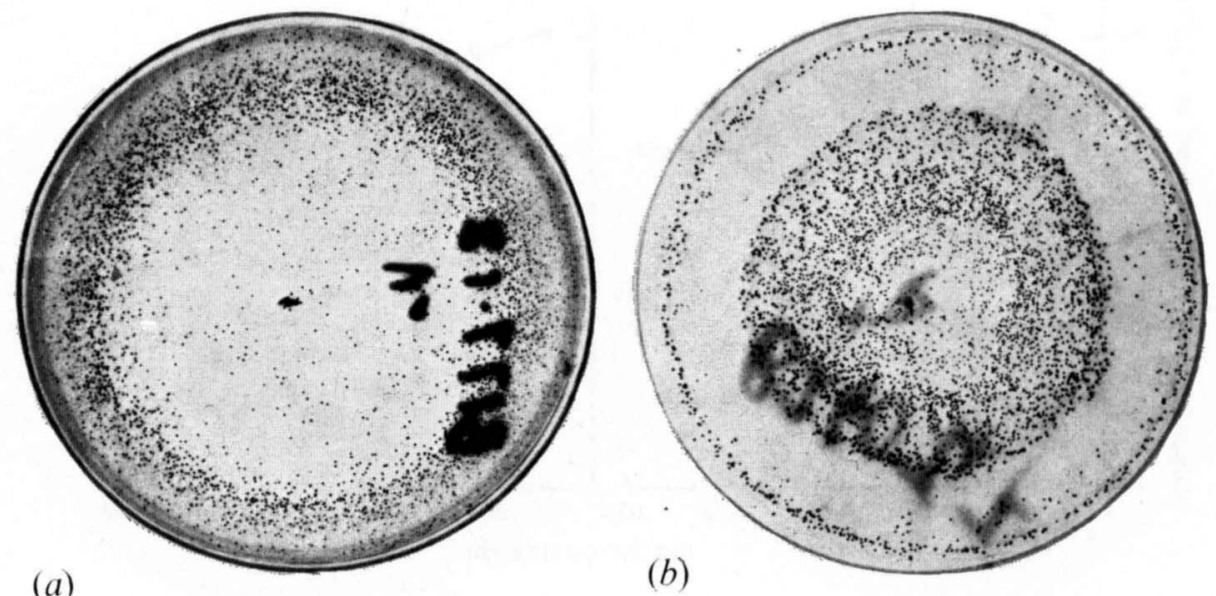

Fig. I. Crossing phenotype of per- $I^{-}$per- $2^{+}$cultures of Sordaria brevicollis grown (a) on corn meal agar; $(b)$ on a sterile dialysis membrane on the surface of corn meal agar.

The existence of a clear pattern of development in a fungal colony poses an interesting problem in differentiation. The fungal colony is a syncytium in which all nuclei are genetically identical and yet the colony is divided into two phenotypically distinct regions. One of the objectives in developing this system is to try to understand how these differentiated regions arise.

\section{Edge effects and protoperithecial development in Sordaria}

The environmental stimuli which result in protoperithecial development in a Petri dish cross were studied. A wild-type culture inoculated in the centre of the dish grew to the edge in about 2 days. During this period it was devoid of protoperithecia: these were only produced in large numbers after it had reached the edge.

Experiments using different sizes of Petri dish suggested that the primary stimulus for making protoperithecia arose when the mycelium reached the edge of the vessel in which the culture was growing. This was true for all fertile cultures regardless of their genotype. Cultures were inoculated into 5, 9 and $\mathrm{I} 4 \mathrm{~cm}$ diameter Petri dishes. In all cases protoperithecial production was apparent only after the cultures had reached the edge of the dish. This effectively eliminated the possibility that either distance from the inoculum or time was the important factor. Experiments were also carried out in which the amount of medium in the Petri dish was varied. Cultures grown on 10,20 and $30 \mathrm{ml}$ media all produced protoperithecia after, and not before, reaching the edge of the plate.

Transfer experiments were conducted in which the culture was grown on a dialysis membrane on the surface of the medium. At different times, the dialysis membrane and culture were removed and the medium re-inoculated. The old medium was perfectly capable of supporting growth, even if a culture had previously been grown on it for 5 days, but the second inoculum, like the first, had to grow to the edge of the plate before protoperithecia were produced. Conversely, cultures were transferred to fresh medium just before they had grown as far as the edge. Again, protoperithecial production coincided with the colony reaching the edge.

These experiments provided no evidence to support the idea that either accumulation of 


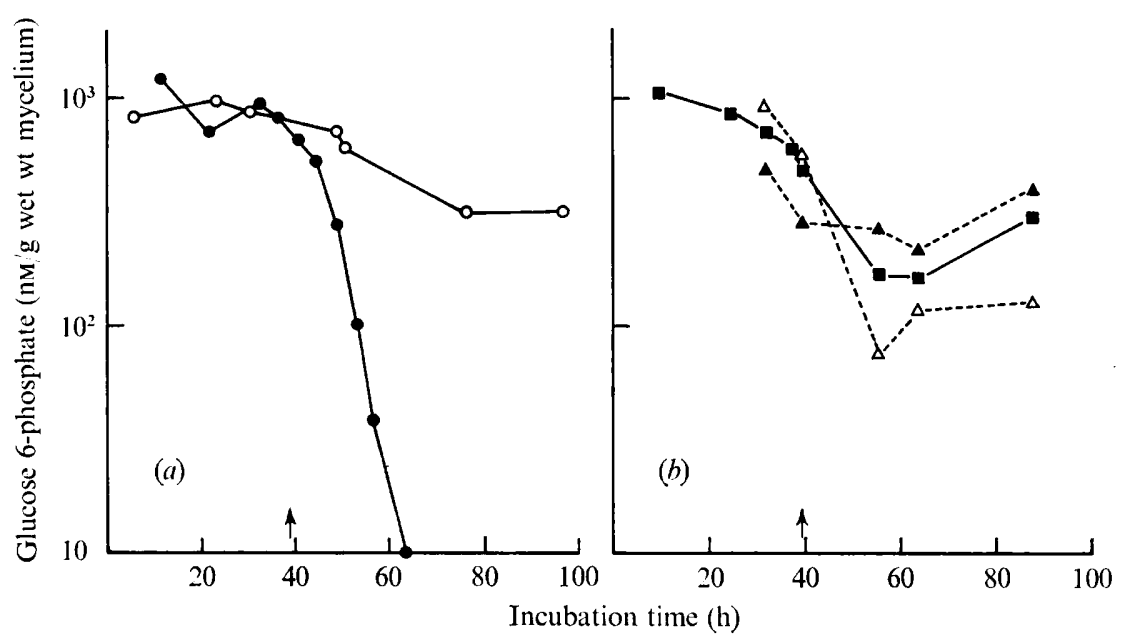

Fig. 2. Glucose 6-phosphate concentration in the mycelium of wild-type and mutant Sordaria brevicollis at various times before and after reaching the edge of the Petri dish. The arrows indicate the time at which the colony reached the edge of the Petri dish. (a) $\bigcirc$, Wild type per $-I^{+}$per- $2^{+}$;

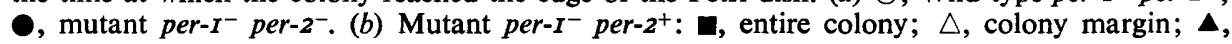
colony interior.

some factor in the medium or exhaustion of a key metabolite was necessary for protoperithecial production. None of the experimental modifications described disrupted the correlation between the time when the colony reached the edge of the plate and protoperithecial production.

\section{Glucose 6-phosphate and protoperithecial production}

Buston \& Rickard (1956) showed that, in Chaetomium globosum, perithecial production was correlated with increases in the concentration of phosphorylated intermediates of sugar metabolism in the medium. Each genotype of Sordaria was analysed by assaying sugar phosphates in the mycelium over several successive days. Figure 2 shows the results of the glucose 6-phosphate assays. Wild-type cultures showed a gradual decline in concentration with time (Fig. 2a). The sterile culture, per-I- per-2-, was strikingly different (Fig. $2 a$ ): when the colony reached the edge of the plate there was a large fall in glucose 6-phosphate concentration. The graphs in Fig. 2 are typical of several independent determinations. The fall in glucose 6-phosphate concentration in the mycelium coincided with the colony reaching the edge when either 14 or $9 \mathrm{~cm}$ diameter dishes were used.

In some experiments the glucose 6-phosphate content of the colony interior and the colony margin was determined separately. For per $-I^{+}$per- $2^{+}$cultures, the interior content was invariably lower than that of the margin, and both showed a slow decline with time. Similarly for per- $I^{-}$per-2- cultures, the sharp fall in glucose 6-phosphate concentration was found in both the inside and the marginal zones (unpublished results).

Convincing evidence for the correlation between glucose 6-phosphate concentration and protoperithecial distribution was provided by the analysis of per- $I^{-}$per- $2^{+}$cultures (Fig. $2 b$ ) The very striking phenotype of this isolate correlated well with the glucose 6-phosphate concentration. Before it reached the edge, a per- $I^{-}$per- $2^{+}$culture had a typical profile with the glucose 6-phosphate concentration lower in the centre than in the colony margin. After reaching the edge, the situation was reversed. The colony interior, where protoperithecial production took place, had a higher glucose 6-phosphate concentration than the sterile 


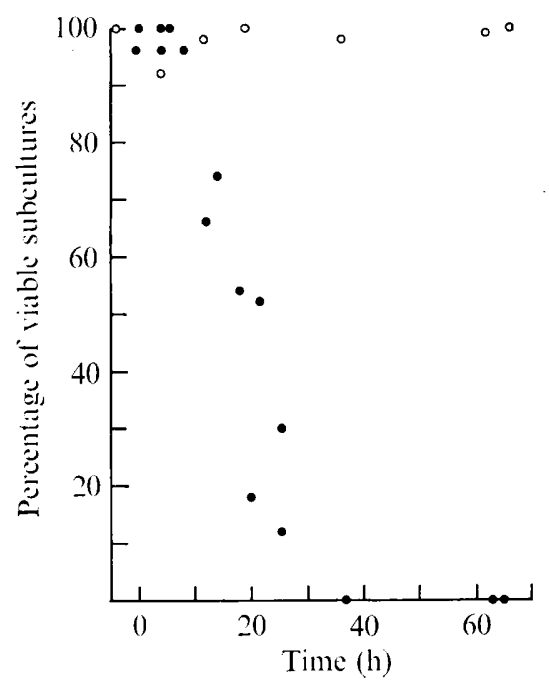

Fig. 3. Viability of per- $I^{-}$per-2- $-(O)$ and per- $I^{+}$per-2 $2^{+}(O)$ cultures as measured by the growth of standardized (I $\mathrm{mm}$ diam) subcultures at various times before and after reaching the edge. Time 0 is the time at which the colony reached the edge of the Petri dish.

margin, which showed a fall similar to, but not as pronounced as that of the per- $I^{-}$per-2cultures.

Buston \& Rickard (1956) suggested that localized increases in sugar phosphate concentration in Chaetomium stimulated the organism to produce protoperithecia. We can find no convincing evidence to support such a causal interpretation. Addition of glucose 6-phosphate to the medium did not stimulate protoperithecial production in Sordaria although such an effect was demonstrated for these compounds by Hawker (1948) in Melanospora destruens. For Sordaria brevicollis, other explanations for the correlation are just as satisfactory.

An apparent paradox exists in that a morphogenetic response occurs on reaching the edge in fertile cultures, whilst the biochemical analysis has revealed an edge effect only in the sterile cultures. The results of subculturing experiments showed that the viability of per-Iper-2- cultures declined after reaching the edge (Fig. 3). As for the glucose 6-phosphate analysis, the relationship of a colony to the plate edge was calculated from its growth rate. Fifty standardized subcultures were taken from each colony using a micropipette (i.d. I $\mathrm{mm}$ ), and the number of these which subsequently grew was recorded. There was a marked drop in viability of cultures of the per- $I^{-}$per-2- genotype which always coincided with reaching the edge.

We are inclined to the view that the fall in the glucose 6-phosphate content of the mycelium is just one indication of an aberrant response to the edge by the per- $I^{-}$per-2- cultures. As a consequence of this abnormal response, the double mutant culture is sterile.

At this stage we do not wish to speculate too much on the nature of the edge effect as it influences either the production of protoperithecia, or their distribution. The experimental approach which we are pursuing is designed to distinguish between two alternatives: first, that the presence of an advancing mycelial front represses protoperithecial production; or sccondly, that there is an induction of protoperithecia when the mycelial front receives the 
correct stimulus. Although we favour the first of these two possibilities, the evidence is not conclusive.

This work has been supported by the Science Research Council. The technical assistance of Miss S. L. Brown and Mr B. J. Doyle is gratefully acknowledged. One of us (D. J. M.) was in receipt of an M.R.C. research studentship. We would like to thank Professor Charlotte Auerbach for her constructive criticism of the manuscript.

\section{REFERENCES}

Buston, H. W. \& Rickard, B. (I956). The effect of a physical barrier on sporulation of Chaetomium globosum. Journal of General Microbiology 15, 194-197.

HAWKER, L. E. (1948). Stimulation of the formation of perithecia of Melanospora destruens by small quantities of certain phosphoric esters of glucose and fructose. Annals of Botany, N.S. 12, 77-79.

HAWKer, L. E. (1966). Environmental influences on reproduction. In The Fungi: An Advanced Treatise, vol. II, pp. 435-469. Edited by G. C. Ainsworth and A. S. Sussman. London: Academic.

InOUE, Y. \& FuRUYA, M. (1970). Perithecial formation in Gelasinospora reticulospora. I. Effects of light at two different growth states. Development, Growth and Differentiation 12, I4I-I 50.

RobInson, W. (1926). The conditions of growth and development of Pyronema confluens Tul. P. omphaloides (Bull) Fuckel. Annals of Botany 40, 245-272. 